\title{
Revisión de cirugía laparoscópica durante la pandemia por COVID-19
}

\section{Laparoscopic surgery during the COVID-19 pandemic a review}

\author{
Jorman H. Tejada MD. 1,2,a, ${ }^{*}$, Jesús H. Tovar MD. ${ }^{1,2, b}$, Diana M. Acosta1,b, Valentina Gutiérrez ${ }^{1, d}$, Alejandra del Pilar Gómez Motta2,e \\ Hospital Universitario Hernando Moncaleano Perdomo. Neiva, Colombia. \\ Universidad Surcolombiana. Neiva, Colombia. \\ Neuroanestesiólogo. Coordinador Servicio de Anestesiología. \\ b Anestesiólogo. \\ c Docente Asistente Posgrado de Anestesiología y Reanimación. \\ d Unidad de Educación Médica. \\ e Médico. Estudiante de Posgrado en Epidemiología.
}

Fuente de financiamiento: Se declara que no existe patrocinio de ninguna entidad.

Declaración conflicto de interés: Los autores no tiene conflictos de interés que reportar, son independientes a instituciones financiadoras y de apoyo. Durante la ejecución del trabajo o la redacción del manuscrito no han incidido intereses o valores distintos a los que tiene la investigación.

Fecha de recepción: 19 de abril de 2021 / Fecha de aceptación: 12 de mayo de 2021

\begin{abstract}
The SARS-CoV-2 pandemic has changed the management protocols of the different surgical areas and practices of health institutions around the world. The countries most affected by the disease have reported an alarming impact on the number of infected and deceased by COVID-19 among health workers. Personnel specialized in airway management have a greater risk of contagion when directly exposed to the aerosolization of the virus. This leads us to consider the probability of postponing or restricting procedures due to limited resources and/or due to patient conditions that increase the risk of death, given that the usual surgical techniques generate more aerosols. This narrative review article aims to analyze the risk of contamination of professionals in laparoscopic surgery of patients infected by COVID-19, proposing strategies to minimize the risk of exposure, identifying the necessary protection measures for health professionals, providing recommendations and adaptations of both the surgical technique and the organization of the operating room according to clinical and scientific evidence.
\end{abstract}

Key words: COVID-19, SARS-CoV-2, job security, laparoscopic surgery.

\section{RESUMEN}

La pandemia por el SARS-CoV-2 ha cambiado los protocolos de manejo de las distintas áreas y prácticas quirúrgicas de las instituciones de salud en el mundo. Los países con mayor afectación de la enfermedad han reportado un impacto alarmante sobre el número de infectados y fallecidos por COVID-19 entre trabajadores de la salud. El personal especializado en el manejo de la vía aérea, posee un mayor riesgo de contagio al exponerse directamente a la aerosolización del virus. Esto conlleva a considerar la probabilidad de posponer o restringir procedimientos por limitación de los recursos y/o por condiciones del paciente que aumenten el riesgo de muerte, dado que las técnicas quirúrgicas habituales generan más aerosoles. Este artículo, de revisión narrativa, pretende analizar el riesgo de contaminación de los profesionales en cirugía laparoscópica de pacientes infectados por COVID-19, proponiendo estrategias para minimizar el riesgo de exposición, identificando las medidas necesarias de protección para los profesionales de la salud, aportando recomendaciones y adaptaciones tanto de la técnica quirúrgica como de la organización del quirófano según la evidencia clínica y científica.

Palabras clave: COVID-19, SARS-CoV-2, seguridad laboral, cirugía laparoscópica. 


\section{Introducción}

os trabajadores de la salud poseen un mayor riesgo ocupacional debido al riesgo de transmisión de múltiples patógenos. La práctica quirúrgica es una de las actividades con mayor riesgo y generación de infecciones asociadas a la atención en salud[1]. El conocimiento de la exposición y contaminación de los profesionales de la salud en cirugía laparoscópica ha sido identificado desde muchos años atrás. Previo a la pandemia, la principal fuente de exposición era el humo quirúrgico producido por el calor del electrobisturí (mono o bipolar), el láser o ultrasónico[2] compuesto por vapor de agua $(95 \%)$, contaminantes inorgánicos $\left(\mathrm{CO}, \mathrm{CO}_{2}\right)$, contaminantes orgánicos (hidrocarburos, ácido hidrociánico y aldehídos), contaminantes biológicos, bacterias y fragmentos de ADN viral[3]. Otra fuente de polución adicional en la cirugía laparoscópica está dada por el aire que se insufla y los aerosoles generados debido al flujo del gas del neumoperitoneo.

Actualmente, la pandemia causada por SARS-CoV-2 reporta que el $80 \%$ de los pacientes tienen síntomas leves o son asintomáticos comportándose como portadores y transmisores potenciales. Las rutas convencionales de transmisión (gotas, aerosoles y contacto directo) son los principales mecanismos involucrados en el contagio, aunque el virus también se ha encontrado en el tracto gastrointestinal, saliva y orina[4]. El centro de control y prevención de enfermedades de China menciona como exposición mayor, la transmisión del virus durante procedimientos médicos de riesgo[5]. Además, el equipo quirúrgico se enfrenta a otra ruta potencial de propagación a través del humo quirúrgico.

Para principios de 2020, China reportó más de 3.300 trabajadores de salud infectados por COVID-19 de los cuales al menos 22 habrían muerto. Por otro lado, en Italia más del 20\% de los infectados corresponden al personal médico y las muertes alcanzaron a más de 50 trabajadores de salud para la misma época. A marzo de 2020, la pandemia se había extendido por todo el mundo con más de 750.000 casos confirmados, incluidas más de 36.000 muertes[4]. Actualmente, el mundo reporta más de 113 millones de contagiados y 2,4 millones de muertes[6]. En Colombia, en abril 8 de 2021 se reportan un total de 2.468.236 casos[7], de los cuales 53.788 casos corresponden al personal de salud; han fallecido 64.524 pacientes, 251 son médicos y enfermeras[8]. Actualmente, se desconoce el número de infecciones nosocomiales atribuidas al proceso de cirugía laparoscópica. Sin embargo, las sociedades científicas internacionales debaten la seguridad de las cirugías mínimamente invasivas por el temor a la transmisión del virus aunque la información de la misma es escasa y las recomendaciones se centran en el control de la aerosolización. Este trabajo busca precisar y analizar el riesgo de contaminación viral durante la laparoscópica en el contexto de la pandemia por COVID-19 de los profesionales sanitarios, así como los medios de protección y prevención necesarios en la práctica clínica.

\section{Métodos}

Se lleva a cabo una revisión narrativa mediante una búsqueda en las bases de datos: Science Direct, PubMed, MedLine, LILACS, Embase, SciELO, Scopus, Google Scholar con el fin de explorar, describir y discutir las medidas de prevención de la aerosolización y el abordaje de pacientes COVID-19 en cirugía laparoscópica considerando los puntos de vista teóricos desde los diferentes tipos de información según la evidencia. Las palabras claves y/o encabezamiento de búsqueda según los términos MeSh fueron: COVID-19, SARS-CoV-2, seguridad laboral, exposición ocupacional, cirugía laparoscópica, y transmisión viral. En el proceso de selección se incluyeron todos los artículos originales con recomendaciones de alto grado de evidencia, publicados en idioma inglés, priorizando aquellos que analizan las medidas de seguridad y manejo de aerosoles en pacientes con diagnóstico confirmado de SARS-CoV-2 Ilevados a cirugía laparoscópica. La revisión de los textos estuvo a cargo de todos los investigadores, los artículos se seleccionaron bajo 3 áreas temáticas preestablecidas; riesgo de contaminación viral, prevención a la exposición de aerosoles, manejo preoperatorio, intraoperatorio y posoperatorio de pacientes contagiados en cirugía laparoscópica. Se revisaron en total 30 artículos en los que el objetivo principal era describir la práctica de la cirugía laparoscópica bajo la pandemia COVID-19, ya que estaban referidos a las áreas temáticas preestablecidas y sirvieron para el desarrollo de la presente revisión. Se estableció un análisis de las recomendaciones adoptadas de acuerdo a la experiencia en un hospital de tercer nivel, los retos de atención y abordaje desde el punto de vista costo-efectivo en el campo de la anestesia y manejo de los pacientes en cirugía laparoscópica.

\section{Resultados}

\section{Riesgo de contaminación viral}

El coronavirus pertenece a la familia Coronaviridae y se clasifica dentro del género de Betacoronavirus, que incluye el coronavirus que causa el SARS-CoV-2 conocido como el síndrome respiratorio del Medio Oriente. El tamaño del virus varía entre 0,06 a 0,14 $\mathrm{mm}$; y posee un ARN monocatenario de $30 \mathrm{~Kb}$ envuelto en sentido positivo[9], con capacidad de infectar distintas especies y huéspedes. Aunque el virus al parecer habita principalmente en el sistema respiratorio, se ha documentado su crecimiento y replicación en muchos tejidos incluido el tracto gastrointestinal. En la fisiopatogenia, la proteína de superficie $S$ del virus se une al receptor de la enzima convertidora de angiotensina II (ACE2) que se encuentra en el tracto gastrointestinal facilitando su replicación[10]. Se ha encontrado ARN viral en heces hasta cinco semanas después de la normalización de las pruebas nasofaríngeas y respiratorias. Sin embargo, no se han encontrado partículas virales infecciosas viables en las heces[11]. A pesar de esta evidencia, se ha especulado en la vía de transmisión fecal-oral teniendo en cuenta las experiencias previas con el virus del síndrome respiratorio agudo severo (SARS) y síndrome respiratorio del Medio Oriente (MERS) pertenecientes a la misma familia. Con certeza se ha demostrado la viabilidad del virus en el aerosol y las superficies duras[12], con duración que varía de horas hasta nueve días, en ciertas superficies[13].

El humo quirúrgico producto de dispositivos generadores de energía, tal como el electro cauterio o bisturí eléctrico, ha sido un elemento de importante riesgo y exposición a múltiples productos químicos, carcinógenos y materiales biológicamente activos como bacterias y virus[3],[5]. Se ha estudiado por más 
de 40 años los riesgos para los trabajadores de la salud[14], reportándose riesgo de infección por aerosolización producida por el virus del papiloma humano[15], la hepatitis B, el Corynebacterium, el $\mathrm{VIH}[16]$ y recientemente por el nuevo SARSCoV-2. La disección o cauterización de los tejidos produce gases que contienen $95 \%$ agua y $5 \%$ de restos celulares. Cerca del $77 \%$ de material particulado dentro del penacho de humo tiene un diámetro promedio de 0,07 $\mu \mathrm{m}$, y las partículas de < $5 \mu \mathrm{m}[17]$ se filtra en las mascarillas quirúrgicas, siendo potencial a ser inhalada por el personal que se encuentra en la sala de cirugía[18]. También, se ha determinado la propagación y la velocidad de viaje del virus en salas y unidades de cuidado intensivo de pacientes con manejo respiratorio entre 1,3 a 5 $\mathrm{m} / \mathrm{s}$, con una distancia de propagación que varía entre 0,6 a 0,8 metros[19],[20]. Ello teniendo en cuenta que se desconoce la dosis infecciosa mínima, y que gran mayoría de quirófanos en Colombia no cuentan con presión negativa.

Se plantean preocupaciones realistas respecto al riesgo del COVID-19 responsable del síndrome respiratorio agudo severo, dada la diseminación en el ambiente durante la cirugía mínimamente invasiva por la aerosolización de partículas a través del neumoperitoneo y la presencia del virus en sangre y heces. La aerosolización viral de hepatitis B por electrocirugía o ultrasonidos se documentó en trabajos dado el desarrollo de la enfermedad[2]. La "Association of perioperative Registered Nurses" (AORN) confirmó la capacidad de esparcimiento del humo causado por el electrocauterio durante las cirugías abiertas con capacidad directa de exposición de todo el personal presente en el quirófano[16]. En los procedimientos laparoscópicos aunque se cuenta con la capacidad de crear entornos cerrados regulados por la entrada y salida de aire definido por los trocares[9], no existe evidencia científica concluyente de la transmisión de COVID-19 por cirugía laparoscópica[21] aunque se asumen debido a los riesgos teóricos de epidemias e infecciones virales en otras patologías.

La evacuación no controlada de neumoperitoneo durante la laparoscopia supone mayor riesgo para el personal y los demás pacientes. Un estudio encontró que posterior al uso de equipos eléctricos o ultrasónicos durante 10 minutos, la concentración de partículas de humo en la cirugía laparoscópica fue significativamente mayor comparado a la cirugía abierta tradicional[22]. Esto ha llevado a plantearse un gran desafío al limitar la realización de procedimientos laparoscópicos a menos que exista un claro beneficio en la disminución de la mortalidad del paciente sobre la cirugía abierta[23].

Aunque la cirugía laparoscópica tiene muchas ventajas como la reducción de la estancia hospitalaria, menor dolor e impacto en los movimientos respiratorio y recuperación rápida en el posoperatorio, también se tiene claro que la proporción de individuos infectados por SARS-CoV-2 asintomáticos no está determinada y son potenciales transmisores de la enfermedad incluyendo a los trabajadores de salud. El riesgo sobre el personal asistencial recae ante los múltiples escenarios de exposición ya que la transmisión se realiza por microgotas, fómites, contacto directo, transmisión fecal-oral y aerosoles, sin excluirse la capacidad del virus en existir sobre superficies inanimadas como el metal, vidrio o plástico hasta nueve días[4], por lo que el equipo quirúrgico y personal de atención en UCI debe propender por la seguridad del paciente y los trabajadores de salud partícipes.

\section{Manejo preoperatorio}

Una vez se evalúa el riesgo y beneficio de ser llevado a cirugía abierta o mínimamente invasiva, se debe verificar la disponibilidad del quirófano con personal humano calificado y capacitado para disminuir el riesgo de transmisión al personal de salud. Definir una ruta hospitalaria dedicada solo al transporte de los pacientes con COVID-19. Involucrar al menor número de personal sanitario y registrarlos para facilitar el seguimiento y contacto. Utilizar todas las medidas de protección personal recomendadas para la prevención de infecciones. Restringir el movimiento de personal dentro y fuera del quirófano. El equipo de anestesia, los computadores, escritorios y máquinas necesarias deben cubrirse con envoltorio plástico para prevenir la contaminación y facilitar su limpieza[4]. Los acondicionadores de aire deben usarse después de la inducción de la anestesia y hasta 20 minutos antes de la extubación[17].

En general, se emplean una serie de estrategias en salas de cirugía para disminuir los riesgos, incluida la ventilación de la sala con presión negativa[17], evitando la contaminación cruzada entre las habitaciones, cambiando el aire del quirófano al menos 12 veces por minuto y en condiciones ideales 25 veces por minuto, minimizar el tiempo y la exposición durante la intubación utilizando mascarilla N95 o máscaras faciales de más alta protección, así como sistemas de evacuación de humo. Se ha planteado la preocupación por las mascarillas N95 ya que filtran partículas de más 0,3 $\mu \mathrm{m}$, dado el pequeño tamaño de partículas generadas por el electrocauterio y bisturí ultrasónico; y el tamaño variable entre 0,06 a 0,14 $\mu \mathrm{m}$ de las moléculas del SARS-CoV-2.

\section{Manejo intraoperatorio}

Los circuitos cerrados de evacuación de humo se utilizan para controlar el penacho quirúrgico, compuesto por dispositivos de succión conectados a la fuente electroquirúrgica. Estudios recientes consideran que el "evacuador de humo" disminuyó el nivel de actividad promedio entre 44,1\% y 99\%, con una eficacia variable debido a la influencia de factores como la velocidad del flujo del evacuador, la angulación del dispositivo quirúrgico desde la piel, la distancia entre la boquilla del evacuador y el sitio quirúrgico, la dirección y velocidad de flujo de aire externo en relación con el flujo de la boquilla. Estos filtros se usan en máquinas de evacuación portátiles, en algunos casos se pueden usar en el sistema de succión de pared para capturar pequeñas cantidades de humo de manera segura. Aunque es recomendado por diferentes organizaciones profesionales que defienden su uso, estas medidas no se utilizan con mucha frecuencia debido al ruido excesivo, al alto costo, los problemas de mantenimiento del equipo, los dispositivos voluminosos y la resistencia del personal de salud.

Los procedimientos laparoscópicos tienen la capacidad de crear un entorno cerrado que permite controlar la entrada y salida de aire a través de los puntos de los trocares que son puntos de acceso bien definidos y se podría especular que podrían reducir el riesgo de transmisión viral al personal del quirófanos a través del humo quirúrgico en comparación con procedimientos abiertos, debido a que se tiene un entorno abdominal cerrado y regulado que puede actuar como barrera de contención. Sin embargo, el proceso constante y dinámico de inserción ex- 
tracción entre los instrumentos laparoscópicos y los puertos de entrada contribuye a una fuga constante de gas insuflado en el quirófano[24]. Cualquier medida que se pueda tomar para reducir la fuga de gas del quirófano debe considerarse a la luz de la pandemia. Según recomendaciones del Colegio de Cirujanos del Reino Unido, una estrategia de mitigación de fugas son los evacuadores de humo utilizados con filtros de aire de partículas ultrabajas (ULPA) con capacidad de purificar el 99,999\% de partículas mayores de 0,1 $\mu \mathrm{m}$ capturando el humo cerca de la fuente de emisión, disminuyendo la exposición del profesional de la salud[25]. La AORN establece que el personal debe instalar filtros ULPA de forma rutinaria para el control del humo quirúrgico. El sistema de evacuación más efectivo es el sistema de triple filtro, que incluye un prefiltro que captura partículas grandes, un filtro ULPA y uno de carbón especial que captura tóxicos encontrados en el humo. Este posee la capacidad de volumen de succión variable para acomodar diferentes niveles de producción de humo[26]. En caso de no contar con este se sugiere el sistema de filtración simple[27], un equipo de bajo costo que cuenta con componentes fácilmente disponibles y de uso inmediato en gran parte de salas quirúrgicas[28].

Todos los cirujanos de procedimientos mínimamente invasivos son conscientes que el trocar es un punto de escape inadvertido de $\mathrm{CO}_{2}$ presurizado del neumoperitoneo, siendo un potencial riesgo el desplazamiento del puerto ante el COVID-19. Se recomienda el uso de puertos de balón que reduce la pérdida inadvertida de presión con fuga en el intercambio de trocar durante la instrumentación, realizar incisiones más pequeñas o disminuir la entrada del trocar en caso de ser más grande con pinzas, reduciendo el riesgo de salida del neumoperitoneo al ambiente de la sala de cirugía[29]. En el caso de no disponer del sistema de evacuación de humo, varios grupos sugieren el uso de succión directa de los trocares de laparoscopia para permitir la evacuación del humo[30], sin embargo, no garantiza alta eficiencia al filtrado, por lo que se recomienda utilizar todos los elementos de protección personal avanzados, incluyendo la mascarilla N95 o mayor.

Es importante considerar las presiones de insuflación y flujo para crear un gradiente de presión cuando dos áreas separadas tienen presiones diferentes. En Norteamérica, la presión intraabdominal promedio utilizada en cirugía laparoscópica es de $15 \mathrm{mmHg}$, comparado con otros lugares a $12 \mathrm{mmHg}$. Se han explorado manejos de presión tan bajas como de $8 \mathrm{mmHg}$ asociado a un bloqueo neuromuscular profundo con adecuado resultado del procedimiento[31]. Los primeros estudios in vivo han sugerido que a presiones intraabdominales altas y tiempos de procedimientos largos contribuyen a un aumento de las tasas de aerosolización, medido por el grado en que los restos celulares se propagan a través de la cavidad abdominal. Dado estos hallazgos, los grupos internacionales recomiendan la realización de procedimientos con flujos bajos (5-10 L/min de $\mathrm{CO}_{2}$ ) y presiones intraperitoneales más bajas entre 8 y $10 \mathrm{mmHg}$ [32].

\section{Manejo posoperatorio}

Una vez terminado el procedimiento, las precauciones de retiro del neumoperitoneo se realiza de forma cuidadosa y controlada aspirando el $\mathrm{CO}_{2}$ intraabdominal, retirando la pieza y los trocares de forma segura. En caso de convertir la cirugía en abierta, se debe tener la precaución de aspirar el $\mathrm{CO}_{2}$ pre- viamente[19]-[33]. Cuando el paciente está en condiciones de extubación, el protocolo a seguir prima en evitar la aerolización del virus durante el procedimiento y realizar la recuperación del paciente en salas previo traslado a la habitación. El personal médico y auxiliar deben retirarse los elementos de protección personal con lista de verificación supervisada para prevenir la contaminación y proceder a la adecuada descontaminación del quirófano siguiendo los protocolos de limpieza y desinfección establecidos en pacientes COVID-19; se sugiere el uso de vaporizadores a base de peróxido de hidrógeno al 0,5\% o etanol al $71 \%$ ya que inactivan eficazmente el virus[4].

\section{Discusión}

Existe preocupación por los trabajadores de la salud ante el riesgo de contagio y muerte ante la pandemia por COVID-19, la exposición en las diferentes áreas de atención y la constante producción de aerosoles por los pacientes. Los procedimientos mínimamente invasivos y el riesgo de transmisión detectada por el humo quirúrgico generan dudas en los servicios de cirugía. Se encuentran posiciones opuestas que inquietan sobre el riesgo/ beneficio para el paciente y el riesgo de exposición a los trabajadores de la salud.

Sin duda alguna el neumoperitoneo y el humo quirúrgico, producen la aerosolización de partículas dentro de las cuales se documentan restos de bacterias y virus[13], en el momento no existe nivel de evidencia que determine que el SARS-CoV-2 se pueda transmitir por la técnica quirúrgica. Sin embargo, otros virus como el del papiloma humano ${ }^{15}$ y hepatitis $B^{2}$, se han encontrado en el ambiente quirúrgico con la capacidad de producir enfermedades ocupacionales, por lo que el uso adecuado de los elementos de protección personal prima en la atención de todos los pacientes.

En marzo de 2020, el consenso de Colegios de Cirugía publicó como recomendación la evaluación de todos los pacientes candidatos a manejo quirúrgico o manejo médico inicial, identificar el beneficio de la cirugía laparoscópica versus abierta, y la exposición del personal de salud. Es importante la comunicación constante del equipo quirúrgico, para determinar la capacidad de elementos mínimos de protección personal, y la idoneidad de las medidas recomendadas en el quirófano para minimizar la exposición al virus[34].

En caso de abordar la técnica laparoscópica, se recomienda disminuir la presión del neumoperitoneo a lo mínimo que sea posible sin comprometer la seguridad del procedimiento, reducir el poder del electrocauterio y la disección con ultrasonido. Al finalizar, utilizar el sistema de aspiración del humo laparoscópico con filtros de partículas, extraer la pieza de tejido después de vaciar el neumoperitoneo por completo al igual que cualquier gasa o mecha utilizada en el procedimiento, y extraer completamente el neumoperitoneo antes de retirar el último trocar[3].

\section{Conclusión}

No existe evidencia científica que soporte la asociación de la realización de procedimientos abiertos o laparoscópicos, y la reducción de transmisión del COVID-19, aunque continua- 
mente se reporta información nueva de la enfermedad y su transmisión. Se entiende como riesgo teórico de transmisión, la aerosolización de virus debido al flujo y pérdida inadvertida del neumoperitoneo que puede exponer al personal de salud. El debate, se centra en si el uso adecuado de las medidas de prevención de la aerosolización pueda ser suficiente para la técnica laparoscópica, ya que el paciente se beneficia al disminuir la estancia hospitalaria y el dolor postoperatorio. Esperamos que se continúen investigaciones concluyentes para establecer toma de decisiones simplificadas.

Agradecimientos: Los autores agradecen al Hospital Hernando Moncaleano Perdomo (Neiva, Colombia) y al personal médico y de enfermería quienes han asumido el rol en línea de frente ante el abordaje de pacientes con COVID-19 en cirugía laparoscópica al aceptar y poner en práctica estas recomendaciones.

\section{Referencias}

1. The Lancet. The Lancet. COVID-19: protecting health-care workers [Internet]. Lancet. 2020;395(10228):922. https://doi. org/10.1016/S0140-6736(20)30644-9.

2. Kwak HD, Kim SH, Seo YS, Song KJ. Detecting hepatitis B virus in surgical smoke emitted during laparoscopic surgery. Occup Environ Med. 2016 Dec;73(12):857-63. https://doi.org/10.1136/ oemed-2016-103724 PMID:27484956

3. Veziant J, Bourdel N, Slim K. Risks of viral contamination in healthcare professionals during laparoscopy in the COVID-19 pandemic. J Visc Surg. 2020 Jun;157(3 3S1):S59-62. https://doi. org/10.1016/j.jviscsurg.2020.04.010 PMID:32340900

4. De Simone B, Chouillard E, Di Saverio S, Pagani L, Sartelli M, Biffl WL, et al. Emergency surgery during the COVID-19 pandemic: what you need to know for practice. Ann R Coll Surg Engl. 2020 May;102(5):323-32. https://doi.org/10.1308/rcsann.2020.0097 PMID:32352836

5. Vourtzoumis P, Alkhamesi N, Elnahas A, Hawel JE, Schlachta C. Operating during COVID-19: is there a risk of viral transmission from surgical smoke during surgery? Can J Surg. 2020 May;63(3):E299-301. https://doi.org/10.1503/cjs.007020 PMID:32449851

6. Covid situation [Internet]. World Health Organization. 2021 [citado 8 abril 2021]. Disponible en: https://covid19.who.int

7. Colombia Situation [Internet]. World Health Organization. 2021 [citado 8 abril 2021]. Disponible en: https://covid19.who.int/region/amro/country/co

8. COVID-19 en Colombia [Internet]. Instituto Nacional de Salud. 2021 [citado 8 abril 2021]. Disponible en: https://www.ins.gov. co/Noticias/Paginas/coronavirus-personal-salud.aspx

9. Birch B, Luo Y, Dhir A. How to minimize airborne droplet contamination while performing laparoscopy in the COVID-19 era. ANZ J Surg. 2020 Dec;90(12):2559-60. https://doi.org/10.1111/ ans.16387 PMID:33022106

10. Wong SH, Lui RN, Sung JJ. Covid-19 and the digestive system. J Gastroenterol Hepatol. 2020 May;35(5):744-8. https://doi. org/10.1111/jgh.15047 PMID:32215956

11. Zhang Wei, Rong-Hui Du, Li Bei, Xiao-Shuang Zheng, Xing-Lou Yang, Ben Hu, Yan-Yi Wang, Geng-Fu Xiao, Bing Yan Z-LS and ZP. Molecular and Serological investigation of 2019-nCOV infec- ted patients: implication of multiple shedding routes. Gastrointest Endosc. 2020;9:0-3.

12. Condon B, Whish-Wilson T, Davis NF, Lawrentschuk N. Implications of COVID-19 on urological laparoscopic surgery. Future Oncol. 2020 Sep;16(26):1941-5. https://doi.org/10.2217/fon2020-0533 PMID:32597203

13. Tran K, Cimon K, Severn M, Pessoa-Silva CL, Conly J. Aerosol generating procedures and risk of transmission of acute respiratory infections to healthcare workers: a systematic review. PLoS One. 2012;7(4):e35797. https://doi.org/10.1371/journal. pone.0035797 PMID:22563403

14. Oh SL, Chia CL, Chen YR, Goo TT, Rao AD, Tan KY, et al. Laparoscopic surgery in a patient with atypical presentation of COVID-19: salient points to reduce the perils of surgery. Singapore Med J. 2020 Aug;61(8):443-4. https://doi.org/10.11622/ smedj.2020086 PMID:32480435

15. Zhou Q, Hu X, Zhou J, Zhao M, Zhu X, Zhu X. Human papillomavirus DNA in surgical smoke during cervical loop electrosurgical excision procedures and its impact on the surgeon. Cancer Manag Res. 2019 Apr;11:3643-54. https://doi.org/10.2147/CMAR. S201975 PMID:31118787

16. Mintz Y, Arezzo A, Boni L, Baldari L, Cassinotti E, Brodie R, et al. The risk of COVID-19 transmission by laparoscopic smoke may be lower than for laparotomy: a narrative review [Internet]. Surg Endosc. 2020 Aug;34(8):3298-305. https://doi.org/10.1007/ s00464-020-07652-y PMID:32458289

17. Gupta N, Agrawal H. COVID-19 and laparoscopic surgeons, the Indian scenario - Perspective [Internet]. Int J Surg. 2020 Jul;79(May):165-7. https://doi.org/10.1016/j.ijsu.2020.05.076 PMID:32479915

18. Limchantra IV, Fong Y, Melstrom KA. Surgical Smoke Exposure in Operating Room Personnel: A Review. JAMA Surg. 2019 Oct;154(10):960-7. https://doi.org/10.1001/jamasurg.2019.2515 PMID:31433468

19. Guo ZD, Wang ZY, Zhang SF, Li X, Li L, Li C, et al. Aerosol and Surface Distribution of Severe Acute Respiratory Syndrome Coronavirus 2 in Hospital Wards, Wuhan, China, 2020. Emerg Infect Dis. 2020 Jul;26(7):1583-91. https://doi.org/10.3201/ eid2607.200885 PMID:32275497

20. Hirota K. Air contamination with SARS-CoV-2 in the operating room [Internet]. J Anesth. 2020;(0123456789):1-4. https://doi. org/10.1007/s00540-019-02660-2 PMID:32562137

21. El M, Ewalds-kvist BM. Since January 2020 Elsevier has created a COVID-19 resource centre with free information in English and Mandarin on the novel coronavirus COVID-19. The COVID-19 resource centre is hosted on Elsevier Connect, the company 's public news and information . 2020;(January).

22. Li Cl, Pai JY, Chen $\mathrm{CH}$. Characterization of smoke generated during the use of surgical knife in laparotomy surgeries [Internet]. J Air Waste Manag Assoc. 2020 Mar;70(3):324-32. https://doi.or g/10.1080/10962247.2020.1717675 PMID:31961784

23. Pawar T, Pokharkar A, Gori J, Pandey D, Rohila J, Dsouza A, et al. The Technique and Justification for Minimally Invasive Surgery in COVID-19 Pandemic: Laparoscopic Anterior Resection for Near Obstructed Rectal Carcinoma. J Laparoendosc Adv Surg Tech A. 2020 May;30(5):485-7. https://doi.org/10.1089/lap.2020.0241 PMID:32315244

24. Uecker JM, Fagerberg A, Ahmad N, Cohen A, Gilkey M, Alembeigi $F$, et al. Stop the leak!: mitigating potential exposure of aerosolized COVID-19 during laparoscopic surgery [Internet]. 
Surg Endosc. 2021 Jan;35(1):493-501. https://doi.org/10.1007/ s00464-020-08006-4 PMID:32974779

25. Chadi SA, Guidolin K, Caycedo-Marulanda A, Sharkawy A, SpineIli A, Quereshy FA, et al. Current Evidence for Minimally Invasive Surgery During the COVID-19 Pandemic and Risk Mitigation Strategies: A Narrative Review. Ann Surg. 2020 Aug;272(2):e118-24. https://doi.org/10.1097/SLA.0000000000004010 PMID:32675513

26. Mowbray NG, Ansell J, Horwood J, Cornish J, Rizkallah P, Parker $A$, et al. Safe management of surgical smoke in the age of COVID-19. Br J Surg. 2020 Oct;107(11):1406-13. https://doi. org/10.1002/bjs.11679 PMID:32363596

27. Zago M, Uranues S, Chiarelli ME, Grandi S, Fumagalli LA, Tavola $M$, et al. Enhancing safety of laparoscopic surgery in COVID-19 era: clinical experience with low-cost filtration devices [Internet]. Eur J Trauma Emerg Surg. 2020 Aug;46(4):731-5. https://doi. org/10.1007/s00068-020-01413-1 PMID:32488448

28. Mintz Y, Arezzo A, Boni L, Chand M, Brodie R, Fingerhut A; and the Technology Committee of the European Association for Endoscopic Surgery. A Low-cost, Safe, and Effective Method for Smoke Evacuation in Laparoscopic Surgery for Suspected Coronavirus Patients. Ann Surg. 2020 Jul;272(1):e7-8. https://doi. org/10.1097/SLA.0000000000003965 PMID:32301809

29. Vigneswaran Y, Prachand VN, Posner MC, Matthews JB, Hussain M. What Is the Appropriate Use of Laparoscopy over Open Procedures in the Current COVID-19 Climate? J Gastrointest Surg.
2020 Jul;24(7):1686-91. https://doi.org/10.1007/s11605-02004592-9 PMID:32285338

30. Patterson TJ, Currie PJ, Beck J, Spence RA, Spence GM. A systematic review of viral transmission risk to healthcare staff comparing laparoscopic and open surgery [Internet]. Surgeon. 2020 Dec;18(6):e72-7. https://doi.org/10.1016/j.surge.2020.06.016 PMID:32690463

31. Neogi $P$, Kumar $P$, Kumar S. Low-pressure Pneumoperitoneum in Laparoscopic Cholecystectomy: A Randomized Controlled Trial. Surg Laparosc Endosc Percutan Tech. 2020 Feb;30(1):30-4. https://doi.org/10.1097/SLE.0000000000000719 PMID:31425453

32. Hanbali N, Herrod P, Patterson J. A safe method to evacuate pneumoperitoneum during laparoscopic surgery in suspected COVID-19 patients. Ann R Coll Surg Engl. 2020 May;102(5):3923. https://doi.org/10.1308/rcsann.2020.0079 PMID:32324055

33. Abras GM. E Silva TAB, Lima LFPN de S, Belangero WD, Lopes MV de R. CO2 filtration during pneumoperitoneum inflation and deflation in patients undergoing laparoscopy during the covid-19 pandemic. Rev Col Bras Cir. 2021;48:1-3. https://doi. org/10.1590/0100-6991e-20202632.

34. de Leeuw RA, Burger NB, Ceccaroni M, Zhang J, Tuynman J, Mabrouk M, et al. COVID-19 and laparoscopic surgery: scoping review of current literature and local expertise. JMIR Public Health Surveill. 2020 Jun;6(2):e18928. https://doi.org/10.2196/18928 PMID:32406853 